Cerebrovascular Diseases 中文目录 2005 年第 19 卷第 6 期

Table of Contents Vol.19, No. 6, 2005

翻译 许剑审校 刘新峰 朱武生 (南京军区南京总医院神经内科, 南京大学医学院)

Translated by Jian Xu and supervised by Xinfeng Liu, Wusheng Zhu, Dept. Neurology, Nanjing

Jinling Hospital, School of Medicine, Nanjing University

目前此翻译内容仅包括自 2004 年以来的《脑血管病》杂志目录。

For the moment, this translation service includes only the table of contents from

CEREBROVASCULAR DISEASES and starts with the first issue of the year 2004.

\title{
述评 Editorial
}

355 已明确需紧急鉴别并处理症状性颈动脉狭窄

The Need for Urgency in Identification and Treatment of Symptomatic Carotid Stenosis Is Already Established

Fairhead, J.F. ; Rothwell, P.M.

Cerebrovasc Dis 2005;19:355-358

\section{会议报告 Meeting Report}

359 欧洲卒中促进会: 卒中暑期学校

European Stroke Initiative: The Stroke Summer School

Brainin, M.

Cerebrovasc Dis 2005;19:359-361

\section{论著 Original Paper}

362 短暂性脑缺血发作患者超急性期弥散加权成像异常预示不可逆性缺血梗死

Hyperacute Diffusion-Weighted Imaging Abnormalities in Transient Ischemic Attack Patients Signify Irreversible Ischemic Infarction

Inatomi, Y. ; Kimura, K. ; Yonehara, T. ; Fujioka, S. ; Uchino, M.

Cerebrovasc Dis 2005;19:362-368

369 由颈动脉张力测量法和手指体积描记法得出的临界关闭压比较

Comparison of Critical Closing Pressures Extracted from Carotid Tonometry and Finger Plethysmography Hsu, H.-Y. ; Chao, A-C. ; Chen, Y.-T. ; Wong, W.-J. ; Chern, C.-M. ; Hsu, L.-C. ; Kuo, J.-S. ; Hu, H.-H. ; Cerebrovasc Dis 2005;19:369-375

376 卒中患者早期出院医疗支持可改善临床结果。这种做法是否亦可减少健康服务及医疗费用?

Early Supported Discharge for Stroke Patients Improves Clinical Outcome. Does It Also Reduce Use of Health Services and Costs?

Fjaertoft, H. ; Indredavik, B. ; Magnussen, J. ; Johnsen, R.

Cerebrovasc Dis 2005;19:376-383

\section{4 抗磷脂抗体阳性卒中患者的临床特点}

Clinical Characteristics of Stroke Patients with Antiphospholipid Antibodies

Terashi, H. ;Uchiyama, S. ; Hashimoto, S. ; Miyazaki, K. ; Tsutsumi, Y. ; Yamazaki, M. ; Iwata, M. 
Cerebrovasc Dis 2005;19:384-390

391 脑梗塞并发结核性脑膜炎

Cerebral Infarcts Complicating Tuberculous Meningitis

Chan, K.H. ; Cheung, R.T.F. ; Lee, R. ; Mak, W. ; Ho, S.L.

Cerebrovasc Dis 2005;19:391-395

\section{回顾及学说 Reviews and Hypotheses}

396 血栓清除能力受损和缺血性卒中：此概念的更多实例及证据

Impaired Washout - Embolism and Ischemic Stroke: Further Examples and Proof of Concept

Sedlaczek, O. ; Caplan, L. ; Hennerici, M. ;

Cerebrovasc Dis 2005;19:396-401

\section{短篇报道 Short Reports}

402 急性卒中时快速抗凝防止缺血性损伤研究-来自学术委员会的最终结果

The Rapid Anticoagulation Prevents Ischemic Damage Study in Acute Stroke - Final Results from the Writing Committee

Chamorro, Á. ; Busse, O. ; Obach, V. ; Toni, D. ; Sandercock, P. ; Reverter, J.C. ; Cervera, A. ; Torres, F. ;

Dávalos, Á. ; for the RAPID Investigators

Cerebrovasc Dis 2005;19:402-404

404 与高分叉颈动脉手术相关的相对于舌下神经的显微外科解剖学标志

Microsurgical Anatomical Landmarks Associated with High Bifurcation Carotid Artery Surgery and Related to Hypoglossal Nerve

Bademci, G. ; Batay, F. ; Vural, E. ; Avci, E. ; Al-Mefty, O. ; Yasaargil, M.G.

Cerebrovasc Dis 2005;19:404-406

\section{病例报告 Case Reports}

407 急性双侧旁正中丘脑梗塞的脑电图可表现为非快动眼睡眠第二阶段样电活动

Acute Bilateral Paramedian Thalamic Infarction Presenting on EEG as Stage 2 Non-REM Sleep

Woerner, J. ; Friolet, R. ; Ventura, F. ; Kardan, R. ; Vuadens, P. ; Arnold, P.

Cerebrovasc Dis 2005;19:407-409

409 仅于晚期得到超声确诊的再发椎动脉夹层

Recurrent Vertebral Artery Dissection with Only Late Ultrasound Confirmation

Morandi, E. ; Anzola, G.P.

Cerebrovasc Dis 2005;19:409-410

410 胼胝体下动脉梗塞引起的遗忘综合征：一种新型的梗死综合征 Amnestic Syndrome of the Subcallosal Artery: A Novel Infarct Syndrome Moussouttas, M. ; Giacino, J. ; Papamitsakis, N.I.H.

Cerebrovasc Dis 2005;19:410-414

\section{给编辑的信 Letter to the Editor}


415 与使用非处方吸入型肾上腺素相关的颖内出血

Intracerebral Hemorrhage Associated with Over-the-Counter Inhaled Epinephrine Cartwright, M.S. ; Reynolds, P.S.

Cerebrovasc Dis 2005;19:415-416

\section{通信 Correspondence}

417 急性卒中的早期颈动脉内膜剥脱术

Early Carotid Endarterectomy in Acute Stroke

Baracchini, C. ; Meneghetti, G. ; Ballotta, E. ;

Cerebrovasc Dis 2005;19:417-418 